\title{
Fuzzy Logic: Theory and Healthcare Application
}

\author{
Hamed Sallam \\ Minnesota State University, Mankato
}

\author{
Afnaan Hashmi \\ Mankato West, Mankato
}

\begin{abstract}
This research was conducted with the intent to apply principles of fuzzy logic to the healthcare setting. This course of action was determined to be ideal because fuzzy logic is well suited to settings with little precision, like the human body and health in general. Fuzzy categories were determined for a variety of health indicators, like cholesterol, blood sugar, blood pressure, and resting heart rate. These same fuzzy categories can be linked to a certain health recommendation which can improve patient health. Hence is the ultimate purpose of the research.
\end{abstract}

Keywords: Fuzzy Logic, Healthcare

\section{INTRODUCTION}

Fuzzy logic is both a new and an old way to process and think about data. It was introduced to modern thought by UC Berkeley's Dr. Lotfi Zadeh in his seminal 1965 paper, "Fuzzy Sets" (Zadeh 1965). Fuzzy logic is a more effective alternative to crisp logic, which was the norm for decades. Crisp logic is all about extremes and dipoles. In crisp logic, it's either zero or one, true or false, or either or. There is no in-between. Fuzzy logic allows for the intermediate value. A fuzzy value can lie between zero and one. For example, in fuzzy logic, something can be $60 \%$ true. In crisp logic, something would have to be either $100 \%$ true, or $0 \%$ true. In this regard, fuzzy logic is much more flexible. A fuzzy item does not have to commit fully to one group, identity, or definition.

Since fuzzy logic mimics the way the human brain works, it's only natural that early cultures had used it extensively. Integral principles of fuzzy logic were developed and used by early Buddhist and Islamic cultures. In fact, this was the norm until Aristotle began championing a crisp and binary approach to the world. This approach was later adopted by the Catholic Church, which spread it throughout Europe. As technology progressed in Europe and America, crisp logic further spread and became very important in machines and systems of all kinds. But, after Zadeh's research and reintroduction, fuzzy logic is spreading and seeing its use increase dramatically (Mathematica Ludibunda).

To better understand fuzzy logic, one must understand the simple sets which make it work. An item is any basic object that is being dealt with. A "set" is group of items with a shared definition or identity (Changing Minds 2018). In a crisp set, the items either fully belong or fully don't. Fuzzy items don't belong to a single set. Rather, they have degrees of membership for each of its sets. A membership degree 
merely states how much an item belongs in a set. An item could be a "full" member with a membership degree of $100 \%$, or it could be a partial member with a degree of $30 \%$. For example, a fuzzy item may have a membership of .35 in set A and .65 in set B. It simultaneously exists in both sets, thus creating an overlap. It should be noted that all of its membership degrees will add up to 1 in the end. This "inbetweenness" is what gives fuzzy logic its unique strengths and abilities.

The ability to exist in multiple sets at once makes fuzzy logic perfectly suited for dealing with natural linguistic inputs. Such natural linguistic inputs that humans might provide may be "very hot", "hot", or "mildly hot". With human language, there are not concrete and discrete tiers separating "levels" of words. This makes it difficult to use crisp logic with linguistic inputs. Crisp logic would require discrete boundaries between tiers. An example of this would be figuring out when something ceases to be 'very hot' in favor of being 'extremely hot'. Fuzzy logic bypasses these definitional dilemmas. Fuzzy logic can arbitrarily define the tiers of words by not having discrete borders. By having membership degrees and overlaps, definitions that resemble the perceived usage definitions can be "created" with fuzzy sets. This then allows for linguistic variables to be used with the system. After all, having a murky or fuzzy border between the tiers can mimic their real usage and perceived meaning.

Fuzzy logic is firmly rooted in the previously mentioned concept of membership degrees. Their value is more apparent when one must have murky borders between a project's inputs. This can be demonstrated with what may be the most iconic image of fuzzy logic: the quintessential trapezoidal membership graph. To understand the fuzzy trapezoidal membership graph, one must first understand the crisp rectangular membership graph. These hypothetical graphs have domains that correspond to the sets. It could be a number-line, but it could also be the tiers of heat in a heat-related problem, or the tiers in any linguistic project. The graph's range is 0 to 1 , thus matching both fuzzy and crisp logic.

The hypothetical crisp graph contains rectangular shapes. At any point in the graph, the y-value must be either 0 or 1 , like every crisp item. There is no state of the in-between. Since these graphs deal with membership values, a y-value of 1 means that the item is fully within a set, while a y-value of 0 means that the item is fully outside of the set. In the crisp graph, the graph line is at 0 when there isn't any membership among whatever is being used. As soon as the item in the domain is a member, the graph immediately jumps all the way up to the maximum of 1 , because it's a full member. This resembles a mathematical step function.

FIGURE 1

\section{CRISP GRAPH FOR TEMPERATURES}
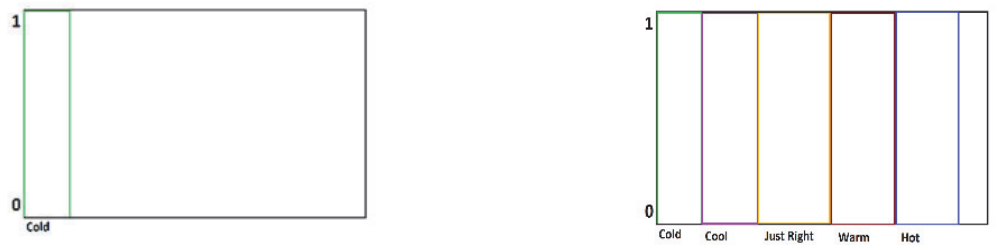

The first crisp graph demonstrates the definition of just one linguistic input, "cold". The green line demonstrates the membership degree of cold along the domain. Any point must reside on the graphed line. And for any point along the domain, there are only two options as to where it can be (on the range). It's either at 0 level, or 1 level, meaning that it's entirely "not cold" or fully "cold". This same idea can be applied to the second graph shown. The second graph introduces more linguistic input categories, each with clear cut demarcations between them.

The fuzzy graph contains trapezoidal shapes. It also has horizonal lines at level 1 when the item is fully within a set. But it offers more flexibility in the transition. Fuzzy logic allows for partial membership degrees. There is no need to have a sudden 'jump' up to 1. Because of this, fuzzy graphs usually have sloped lines leading to and from the full membership horizontal lines. This gives rise to the 
trapezoidal shape, and reflects fuzzy logic's greater flexibility, greater accuracy, and greater realism compared to crisp logic. It should be noted that a fuzzy membership graph is not required to have trapezoidal shapes, they may also be triangles with pointed tops at level 1 .

The following diagram shows what a fuzzy membership graph could look like when dealing with linguistic temperature levels and their perceived linguistic boundaries. In accordance with fuzzy principles, any item (temperature) has varying levels of membership in multiple categories (cold, cool, etc.).

\section{FIGURE 2 \\ FUZZY GRAPH FOR TEMPERATURES}

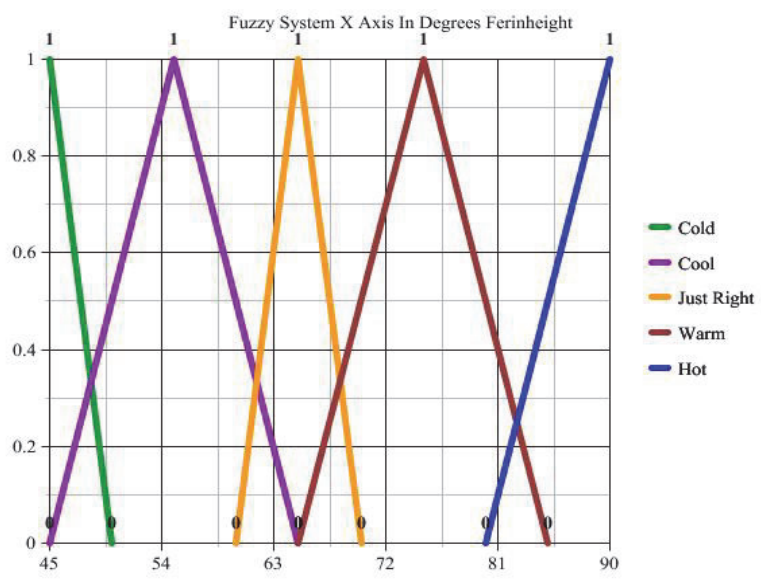

(Williams)

An important consideration that must be taken into account when dealing with any fuzzy application is the fuzzy logic context diagram. An understanding of the flow of data is essential to creating and building upon any fuzzy application.

In any data system, the first thing that must be dealt with is the input data. For a fuzzy application, this input is usually raw numerical values or linguistic inputs. This input data then goes through the process of 'fuzzification'. This raw input gets assigned its various membership degrees in this step, thus making it a fuzzy value. This newly fuzzy value then gets run against the pre-set rules of the system to determine the optimum value or course of action. This result then gets 'defuzzified', and then is used to execute what has been determined to be the proper course of action.

The following diagram presents a fuzzy controller for a generic contained system. The data from the core process is collected and fuzzified. Once fuzzy, it will be run against the system's rules to determine the next course of action for the process. But before the new plan can get set in motion, it must be defuzzified and applied towards the process.

For example, this process can represent a fuzzy HVAC system. The process is whatever action the HVAC system is executing, heating, cooling, or nothing. The rules may be simple like "if it is hot, then cool", or "if it is cold, then heat". With this information in mind, the context diagram can be interpreted as follows:

First, the fuzzy system will measure the temperature of the environment. This raw temperature will then be fuzzified and assigned a new value. In this example, a temperature of 80 degrees Fahrenheit might be fuzzified and become "very hot". And then based on the system's rules, this fuzzy value will result in a command of "lower the temperature". This would then have to be defuzzified and promptly applied to the HVAC system. In the end, the usage of a fuzzy controller can result in a controlled and "ideal" environment for whatever system or functionality it is applied to. 
FIGURE 3

FUZZY CONTROLLER DIAGRAM

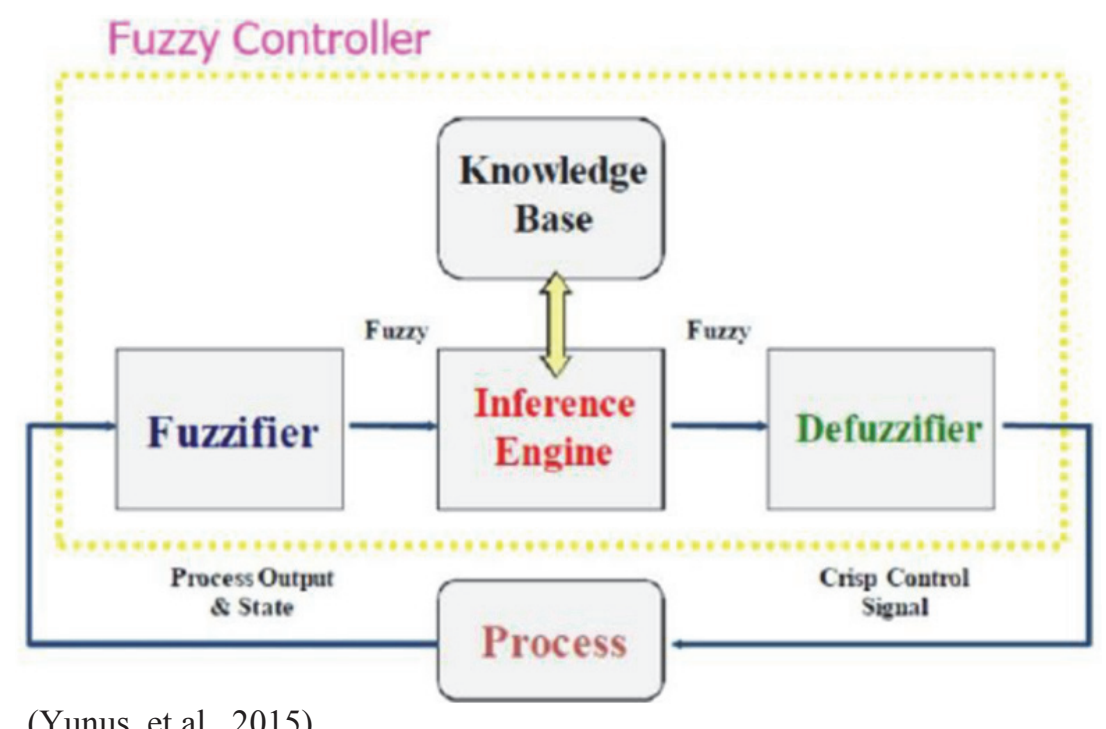

(Yunus, et.al., 2015)

To encapsulate what makes fuzzy logic so special, it is its ability to achieve "imprecise precision".

\section{REAL APPLICATIONS OF FUZZY LOGIC}

Such a powerful logic system has the ability to upend and revolutionize a plethora of existing industries. Sundry real-life examples will be examined, but a special focus will be provided to a certain healthcare application of fuzzy logic. Indeed, fuzzy logic already has changed the face of a number of fields and industries because of the added flexibility and inherent problem-solving strength that comes from fuzzy logic's imprecise precision. It should be noted, that almost all of the world's industries were designed or created by humans. As a result, they either purposely or accidentally mimic human behavior and thought processes. These mimicries can be found in many aspects of any individual field. Because of this intrinsic design theme, fuzzy logic can work astoundingly well in these same fields. Fuzzy logic expressly mimics the natural human way of thinking; thus directly complementing these industries.

Traces of fuzzy logic can be found in any number of industrial applications. Fuzzy control systems have increased the efficiency of industries like cement manufacturing, steel production, shipping, turbine control, and engine maintenance to name a few. Fuzzy logic has also made its way into infrastructure projects, like subway systems, steam turbine optimizations, and power supply control among others. The added benefit of fuzzy logic in these industries come from the control that fuzzy logic grants these systems. In a traditional "crisp" subway system, a train will stop completely if there is another train closer to the shared junction. The strict and sharp rule not only limits the efficiency of the endeavor, but also has higher operating costs because more fuel is expended than needed when the trains stop and lose momentum, and then start up and regain lost momentum. In Japan's "fuzzy" subway system, when two trains both approach a shared junction, the fuzzy rules slow the appropriate train down just the right amount based on the other's proximity, so that as soon as one train leaves the junction the other is entering. This is not only a major time saver, but also a fuel saver (Kosko et. Al. 1993).

Another famous example of fuzzy logic being used to solve an infrastructure problem was that involving a Greek railroad. A research team used a fuzzy logic controller to discover defects in the wheels of the trains. They did so by analyzing recordings of the train wheel's vibrations. To make the data significant, the team recorded vibrations from both "healthy", fully functioning wheels and defective wheels. The gathered data was henceforth "fuzzified" and run against a series of rules (see 4. Illustrative Examples). In this controller, the vibrational inputs were converted into damage reports, and then based 
on how damaged the wheels were, the controller outputted how much maintenance should be done on the wheel (Skarlatos et al. 2004).

The benefits and applications of fuzzy logic aren't limited to mechanical systems. Fuzzy logic models can be used to solve a number of abstract problems through the use of appropriate fuzzy software. Three of these problems that can be solved with fuzzy logic will be detailed later in the text.

\section{A FUZZY TIPPING PROBLEM}

To paint a better picture of the power of fuzzy logic, a specific application will be brought to attention and detailed. This application affects the hospitality sector. It parlays fuzzy logic to achieve a costeffective and optimal solution to the problem at hand. After all, the imprecise precision of fuzzy logic makes it well suited for solving multi-variable problems.

The real-world application that will be analyzed seeks to answer the age-old question, "How much to tip the waiter?" SciKit Fuzzy's fuzzy program for determining tip amounts will be analyzed. Keeping with fuzzy tradition, the situation's inputs, outputs, and rules must be clearly established before diving into code.

The inputs for this fuzzy problem are the same factors that a restaurant patron would take into account to determine the tip amount. These are: service quality and food quality. Fuzzy logic's strength become readily apparent when the question of how to input the values comes up. A crisp system would have someone assigning arbitrary numerical ratings for each of the inputs. In the fuzzy system, natural linguistic inputs can be used. An example of these inputs are: "very bad", "bad", "average", "good", and "great". They not only match natural human language used when describing the quality of something, but also match human language's murky definitional borders because of fuzzy logic's practice of overlaps and membership degrees.

\section{FIGURE 4 \\ FUZZY FOOD QUALITY GRAPH}

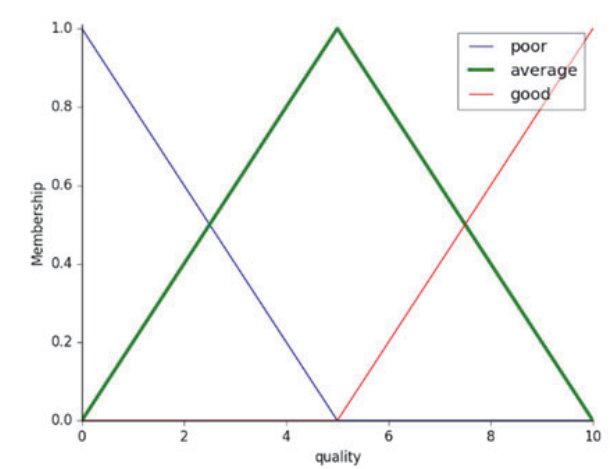

(SciKit Fuzzy, 2018)

This diagram shows the fuzzy membership graph that the three inputs use (the diagram shows food quality specifically, however the same slopes and membership values were applied to the other inputs).

The output in this example is the percentage amount tipped. Scikit Fuzzy assigned only three rules to this problem, though the rule number and complexity can be increased if the situation requires it. The rules are:

Rule 1: If the service was good or the food was good, then the tip will be high.

Rule 2: If the service was average, then the tip will be medium.

Rule 3: If the service was poor and the food was poor, then the tip will be low.

(SciKit Fuzzy 2018) 
To start programing this tipping program, the appropriate libraries must be imported. Scikit Fuzzy import 'numpy', 'skfuzzy', and 'control'. Using the 'control' library's 'antecedent' function, variables for service quality, food quality, and tip amount can all be initialized. A range of numerical percentage values is then assigned to the separate 'sets' of 'tips'. Scikit fuzzy had a 'low tip subset', a 'medium tip subset', and a 'high tip subset'. Using Python's built in operators for 'and' and 'or', the three rules can be formally programmed into existence. Then, a control system can be created using the 'control' library's 'ControlSystem' function with the three rules as parameters. The same library's 'ControlSystemSimulation' function can then be called with the prior control system as a parameter. This 'runs' the programmed rules for a specific set of inputs and then outputs the suggested tip percentage after a period of defuzzification.

\section{HEALTHCARE APPLICATION}

Fuzzy logic and its associated functionality can be applied to sundry health care applications. An interesting hypothetical application that will be examined is one that would greatly improve the knowledge that a 'layperson' has over his/her current medical condition. Such an application has great promise and a potentially massive effect on the lives of its users.

The modern state of healthcare today consists of inefficiencies (long lines, queues, etc.), lackluster insurance coverage and availability, and patient apathy. All of these factors contribute to a weakened healthcare system which ultimately hinders patients' efforts to protect their health and worsens a society's overall health. However, a fuzzy logic healthcare application can put more power in the hands of patients and demonstrate to them the urgency of seeking treatment.

There are many opportunities for patients to become aware of their standard vitals, which can include resting heart rate, blood pressure, cholesterol, etc. These elements and other unlisted elements can function as indicators of a larger, more serious health problem. Enter fuzzy logic. By assigning fuzzy categories to different values of a certain vital sign, one can end up with a fuzzy rules database. This fuzzy rules database can act upon a given set of inputted vital signs to deliver relevant health advice towards the layman user. Some possible range examples can be found below for certain vital signs.

To demonstrate this, a hypothetical fuzzy algorithm will be applied to Table 1. Table 1 presents fuzzy ranges and categories that pertain to one's blood pressure. The normal category lists a systolic blood pressure of "less than 120 " as a prerequisite. Since the data is fuzzy, this range demonstrates where the blood pressure would be majority "normal". Just life the fuzzy tipping problem and any fuzzy logic program, a set of rules must be established. These rules are vital to assigning an input to a group and then acting based on which group(s) the input belongs to. Some rules that may guide diagnosis for one's blood pressure and identify the input are as follows:

1. IF systolic blood pressure is less than 120 AND diastolic is less than 80 THEN blood pressure is mostly normal.

2. IF systolic blood pressure is 120-129 AND diastolic is less than 80 THEN blood pressure is elevated.

3. IF systolic blood pressure is 130-139 AND diastolic is 80-89 THEN blood pressure is indicative of moderate hypertension.

4. IF systolic blood pressure is 140 or higher OR diastolic is 90 or higher THEN blood pressure is indicative of serious hypertension.

5. IF systolic blood pressure is 180 or higher OR diastolic is 120 or higher THEN blood pressure is indicative of emergency hypertension.

Each of these rules will trigger an individualized response that contains tailored advice relevant to the user. For example, if one were to have a systolic blood pressure of $145 \mathrm{~mm} \mathrm{Hg}$ and a diastolic blood pressure of $125 \mathrm{~mm} \mathrm{Hg}$, the fuzzy rules database would diagnose the person with some mixture of serious and emergency hypertension. This would result in advice imploring the user to immediately go to his doctor and seek professional medical treatment. The same process can be applied to other variables. These individual vital sign values will each run against their corresponding rules (which correspond to the 
data shown in the below tables) and will result in a mixture of individualized and overall advice being given to the user.

These values were ascertained through a multitude of respectable health sources. A finished product could have different values, more refined values, or values that are in accordance with the guidelines set out by a specific organization. Table 1 deals with ranges and categories for one's blood pressure (systolic and diastolic). Table 2 presents similar ranges but for one's cholesterol level. Table 3 presents potential ranges for one's resting blood sugar level, and Table 4 does the same for diabetic blood sugar levels. Tables 5 and 6 present the resting heart rate levels for men and women respectively.

This process works well with the data gathered by a medical professional, but it is not limited to it. Some people who must monitor their health through vital sign measurements can use this fuzzy application to help prompt them into initiating an emergency checkup. If a normally apathetic patient finds that their health might be at risk, they too would be motivated to schedule a checkup with their medical provider. In addition, if a user of the service lacks health insurance and must choose their checkups wisely, this fuzzy application can help guide them in a general direction. It must be noted, however, that this service is in no way competing with medical professionals or replacing them. It is only intended to be used as a supplementary guide for the patient and possibly the medical professional.

TABLE 1

\section{BLOOD PRESSURE CATEGORIES}

\begin{tabular}{|l|l|l|l|}
\hline Fuzzy Attributes & Systolic $(\mathrm{mm} \mathrm{Hg})$ & & Diastolic $(\mathrm{mm} \mathrm{Hg})$ \\
\hline Normal & Less than 120 & And & Less than 80 \\
\hline Elevated & $120-131$ & And & Less than 80 \\
\hline Moderate Hypertension & $129-141$ & Or & $80-91$ \\
\hline Serious Hypertension & 140 or higher & Or & 89 or higher \\
\hline Emergency Hypertension & Higher than 180 & And /Or & Higher than 120 \\
\hline
\end{tabular}

TABLE 2

CHOLESTEROL CATEGORIES

\begin{tabular}{|l|l|l|l|l|}
\hline Fuzzy Attributes & $\begin{array}{l}\text { Total Cholesterol } \\
(\mathrm{mg} / \mathrm{dl})\end{array}$ & $\begin{array}{l}\text { HDL Cholesterol } \\
(\mathrm{mg} / \mathrm{dl})\end{array}$ & $\begin{array}{l}\text { LDL Cholesterol } \\
(\mathrm{mg} / \mathrm{dl})\end{array}$ & Triglycerides \\
\hline Desirable & Less than 200 & 60 or higher & Less than 100 & Less than 149 \\
\hline $\begin{array}{l}\text { Moderately } \\
\text { Elevated }\end{array}$ & $200-242$ & $38-62$ & $98-162$ & $148-202$ \\
\hline $\begin{array}{l}\text { Dangerously } \\
\text { Elevated }\end{array}$ & 238 or higher & Less than 41 & $158-192$ & 200 or higher \\
\hline Emergency & & & 190 or higher & \\
\hline
\end{tabular}


TABLE 3

FASTING BLOOD SUGAR CATEGORIES

\begin{tabular}{|l|l|}
\hline Fuzzy Attributes & Fasting Blood Sugar Level (mg/dL) \\
\hline Dangerously Low-Emergency & 50 or below \\
\hline Acceptable & $70-92$ \\
\hline Ideal & $88-112$ \\
\hline Pre-diabetic (Moderately high) & $108-132$ \\
\hline Diabetic (High) & $128-162$ \\
\hline Very high & $157-243$ \\
\hline Dangerously high-Emergency & $238-302$ \\
\hline Warning-Immediate medical help & 300 or above \\
\hline
\end{tabular}

TABLE 4

DIABETIC BLOOD SUGAR CATEGORIES

\begin{tabular}{|l|l|l|}
\hline Fuzzy Attribute & Before Meal (mg/dL) & Two Hours After Meal (mg/dL) \\
\hline Excellent & $72-109$ & $90-128$ \\
\hline Good & $108-146$ & $126-182$ \\
\hline Acceptable & $145-182$ & $180-236$ \\
\hline Dangerous & 180 or above & 234 or above \\
\hline
\end{tabular}

TABLE 5

RESTING HEART RATE CATEGORIES MALE

\begin{tabular}{|l|l|l|l|l|l|l|l|}
\hline Fuzzy Attributes & Super & Excellent & Good & $\begin{array}{l}\text { Above } \\
\text { Average }\end{array}$ & Average & $\begin{array}{l}\text { Below } \\
\text { Average }\end{array}$ & Poor \\
\hline $\begin{array}{l}\text { Heart rate for 18- } \\
25 \text { years old }\end{array}$ & $49-57$ & $53-63$ & $59-68$ & $64-72$ & $68-76$ & $72-84$ & $82+$ \\
\hline $\begin{array}{l}\text { Heart rate for 26- } \\
35 \text { years old }\end{array}$ & $49-57$ & $53-63$ & $60-67$ & $64-72$ & $69-76$ & $73-84$ & $82+$ \\
\hline $\begin{array}{l}\text { Heart rate for 36- } \\
\text { 45 years old }\end{array}$ & $50-58$ & $55-64$ & $61-68$ & $65-72$ & $69-77$ & $74-85$ & $83+$ \\
\hline $\begin{array}{l}\text { Heart rate for 46- } \\
55 \text { years old }\end{array}$ & $50-59$ & $56-64$ & $63-69$ & $66-73$ & $70-78$ & $75-85$ & $84+$ \\
\hline $\begin{array}{l}\text { Heart rate for 56- } \\
65 \text { years old }\end{array}$ & $51-58$ & $55-63$ & $60-69$ & $66-73$ & $70-77$ & $74-83$ & $82+$ \\
\hline $\begin{array}{l}\text { Heart rate for } \\
65+\text { years old }\end{array}$ & $50-57$ & $54-63$ & $60-67$ & $64-71$ & $68-75$ & $72-81$ & $80+$ \\
\hline
\end{tabular}


TABLE 6

RESTING HEART RATE CATEGORIES FEMALE

\begin{tabular}{|l|l|l|l|l|l|l|l|}
\hline Fuzzy Attributes & Super & Excellent & Good & $\begin{array}{l}\text { Above } \\
\text { Average }\end{array}$ & Average & $\begin{array}{l}\text { Below } \\
\text { Average }\end{array}$ & Poor \\
\hline $\begin{array}{l}\text { Heart rate for 18- } \\
25 \text { years old }\end{array}$ & $54-60$ & $61-65$ & $66-69$ & $70-73$ & $74-78$ & $79-84$ & $85+$ \\
\hline $\begin{array}{l}\text { Heart rate for 26- } \\
35 \text { years old }\end{array}$ & $54-59$ & $60-64$ & $65-68$ & $69-72$ & $73-76$ & $77-82$ & $83+$ \\
\hline $\begin{array}{l}\text { Heart rate for 36- } \\
45 \text { years old }\end{array}$ & $54-59$ & $60-64$ & $65-69$ & $70-73$ & $74-78$ & $79-84$ & $85+$ \\
\hline $\begin{array}{l}\text { Heart rate for 46- } \\
55 \text { years old }\end{array}$ & $54-60$ & $61-65$ & $66-69$ & $70-73$ & $74-77$ & $78-83$ & $84+$ \\
\hline $\begin{array}{l}\text { Heart rate for 56- } \\
65 \text { years old }\end{array}$ & $54-59$ & $60-64$ & $65-68$ & $69-73$ & $74-77$ & $78-83$ & $84+$ \\
\hline $\begin{array}{l}\text { Heart rate for } \\
65+\text { years old }\end{array}$ & $54-59$ & $60-64$ & $65-68$ & $69-72$ & $73-76$ & $77-84$ & $84+$ \\
\hline
\end{tabular}

\section{CONCLUSION}

Fuzzy logic truly is a powerful and radically new way of thinking and dealing with problems. As has been shown, it can usurp existing methodologies in sundry industries and fields and then greatly increase the efficiency of those fields. By assigning each individual item membership degrees in different sets, thus allowing for overlaps between sets, more advanced problems can be solved with greater accuracy. Fuzzy logic has already seen the light of day in public transportation, most industrial applications, and any enterprise where efficiency is key. However, massive benefits are to be had when fuzzy logic is applied to the healthcare industry.

By applying fuzzy logic to healthcare related issues such as diagnosis, both medical professionals and everyday people can have a better sense of their health. From a holistic, societal standpoint, such a development is beneficial for the increased knowledge and independence it affords the populace. A fuzzy healthcare program that mirrors the one previously described in this paper can increase the efficiency of the healthcare system as a whole by streamlining the diagnosis process. It empowers those who lack sophisticated medical knowledge. It can also increase the number of people who regularly check their health by directly informing them of the need to consult with a medical professional.

In the future, a mobile app can be created that has all the functionality of the described fuzzy healthcare diagnosis program. However, technology is constantly changing, and the fuzzy program need not be constrained by any specific format. As wearable technologies not only improve in their sensing ability but also increase in their ubiquity, the fuzzy program can be made part of such devices. This would result in an even faster diagnosis, and one that's even more accessible to the user. Since the wearable can collect the vital sign data of the person, he could avoid mundane trips to a doctor to collect the same data. This would increase the efficiency of hospitals because they could get away with less time and financial expenditure on minor tests. In addition, if medical professionals or organization fully embrace fuzzy diagnosis programs their accuracy and added benefits to the sector would both increase dramatically. The strengths of fuzzy logic are uniquely predisposed to the medical sector, and fuzzy diagnosis programs significantly benefit everyone involved. 


\section{REFERENCES}

Bělohlávek, R., \& Klir, G. (2011). Concepts and Fuzzy Logic. MIT Press.

Bonissone, P.P., Badami, V., Chiang, K.H., Khedkar, P.S., Marcelle, K.W., \& Schutten, M.J. (n.d.). Industrial Applications of Fuzzy Logic at General Electric - IEEE Journals \& Magazine.” Design and Implementation of Autonomous Vehicle Valet Parking System - IEEE Conference Publication. Wiley-IEEE Press. Retrieved from ieeexplore.ieee.org/document/364490/.

Dingle, N. (2011, November 4). Artificial Intelligence: Fuzzy Logic Explained. Next-Generation Control Engineer Advice | Control Engineering. Retrieved from https://www.controleng.com/articles/artificial-intelligence-fuzzy-logic-explained/

Fuzzy Control Systems: The Tipping Problem. (n.d.). Welcome to PyDAQmx's Documentation! PyDAQmx 1.0.0 Documentation. Retrieved from pythonhosted.org/scikitfuzzy/auto_examples/plot_tipping_problem_newapi.html.

History and Objections. (n.d.). Fuzzy Logic. Retrieved from www.calvin.edu/ pribeiro/othrlnks/Fuzzy/history.htm.

Huizen, J. (2018). Blood sugar chart: Target levels throughout the day. Retrieved from https://www.medicalnewstoday.com/articles/317536.php

Introduction. (n.d.). Fuzzy Logic Example \#2. Retrieved from petro.tanrei.ca/fuzzylogic/fuzzy_mehaan_joy.html.

Kosko, B., \& Isaka, S. (1993). Fuzzy Logic. Calvin College Engineering Department, Scientific American. Retrieved from www.calvin.edu/ pribeiro/othrlnks/Fuzzy/files/Scientific\%20American.pdf.

Larsen, P.M. (2008, August). Industrial Applications of Fuzzy Logic Control. Egyptian Journal of Medical Human Genetics, Elsevier, 20. Retrieved from www.sciencedirect.com/science/article/pii/S0020737380800502.

Lifespanfitness. (n.d.). Your Resting Heart Rate: What Is Normal and Healthy? Retrieved from https://www.lifespanfitness.com/fitness/resources/articles/your-resting-heart-rate-what-is-normaland-healthy

McNeill, D., \& Freiberger, P. (1994). Fuzzy Logic Revolutionary Computer Technology That Is Changing Our World. Simon \& Schuster.

Meehan, G., \& Joy, M. (1998). Animated fuzzy logic. Journal of functional Programming, 8(5), 503-525. Pythagorean Theorem - History. (n.d.). Fuzzy Logic - History. Mathematica Ludibunda. Retrieved from mathematica.ludibunda.ch/fuzzy-logic7.html.

SciKit Fuzzy. (2018). Fuzzy Control Systems: The Tipping Problem.

Set Theory. (2018). Changing Minds. Retrieved from changingminds.org/disciplines/argument/syllogisms/set_theory.htm.

Skarlatos, D., Karaksis, K., \& Trochidis, A. (2004). RAILWAY WHEEL FAULT DIAGNOSIS USING A FUZZY-LOGIC METHOD. The National Academies of Sciences Engineering Medicine, Elsevier. Retrieved from trid.trb.org/view/745336

Understanding Blood Pressure Readings. (n.d.). Retrieved from http://www.heart.org/en/healthtopics/high-blood-pressure/understanding-blood-pressure-readings

What Are the Recommended Cholesterol Levels by Age? (n.d.). Retrieved from https://www.healthline.com/health/high-cholesterol/levels-by-age

Williams, Z. (n.d.). Multi-Core Systems. Fuzzy Logic and Fuzzy Systems. Retrieved from www.cs.uaf.edu/2012/fall/cs441/students/zw_fuzzy/.

Yunus, M.A.M., Zakariah, A., Faramarzi, M., \& Jamian, J.J. (2015). Medium size dual-axis solar tracking system with sunlight intensity comparison method and fuzzy logic implementation. ResearchGate.net

Zadeh, L. (2004). Fuzzy Sets. Egyptian Journal of Medical Human Genetics, Elsevier. Retrieved from www.sciencedirect.com/science/article/pii/S001999586590241X. 